\title{
ESTHESIONEUROBLASTOMA: A CASE REPORT
}

Manish Kumar Sachan'1, A. K. Jain², Freni J. K³, Kavish Jhawar ${ }^{4}$

\section{HOW TO CITE THIS ARTICLE:}

Manish Kumar Sachan, A. K. Jain, Freni J. K, Kavish Jhawar. "Esthesioneuroblastoma: A Case Report”. Journal of Evolution of Medical and Dental Sciences 2014; Vol. 3, Issue 25, June 23; Page: 6870-6873,

DOI: $10.14260 /$ jemds/2014/2830

ABSTRACT Olfactory neuroblastoma is an uncommon neuroectodermal tumour. Its biological activity ranges from indolent growth to local recurrence and rapid wide spread metastasis. Treatment option consists of surgical resection followed by radiation therapy for primary lesion and addition of chemotherapy for advanced, recurrent or metastatic lesion.

KEYWORDS: Esthesioneuroblastoma, Olfactory neuroblastoma, Nasal cavity.

INTRODUCTION: Olfactory neuroblastoma is a tumour of neural crest origin arising from the olfactory neuroepithelium of the roof of the nasal cavity and paranasal sinuses. It was first described by Berger and Luc in the French medical literature. Less than 1000 cases have been described in literature since 1924. Olfactory neuroblastoma represents 5-10\% of sinonasal tumours and less than $1 \%$ of all malignant tumours and has no sex predilection. It has bimodal age distribution occurring most commonly in teenagers and in sixth decade of life. ${ }^{1}$

The symptoms of olfactory neuroblastoma include unilateral progressive nasal obstruction, rhinorrhoea, epistaxis, anosmia and headache. Extension of disease into orbit may result in proptosis, diplopia and retrobulbar pain. However, patient often presents with non specific symptoms resembling chronic sinusitis and therefore many cases are diagnosed late with advanced disease. ${ }^{1}$ Cervical lymph node metastasis is most frequent but late( 6 to $20 \%$ cases). Distant metastasis is seen in 9 to $13 \%$ of patients and tends to involve bone, lungs and liver. Local recurrence is relatively frequent (2-3years after initial treatment).

It usually grows slowly but with locally invasive behavior. It spreads through submucosal planes to the surrounding structures and involves anterior cranial fossa destroying the cribriform plate with propagation along the perineural spaces of olfactory nerves. Orbital extension takes place through erosion of lamina papyracea.

Nasal endoscopy usually confirms an exophytic polypoid or sessile mass arising from the superior portion of the nasal cavity. ${ }^{1}$

This tumour which can some times present with intracranial symptoms is relatively slow growing and is considered to be radiosensitive. Surgery followed by radiation therapy is the preferred treatment. ${ }^{2}$

CASE REPORT: A 35 year old male presented to us with complaints of mass in left nasal cavity, nasal obstruction, nasal discharge, headache, inability to close both eyes and occasional nasal bleeding of 15 days duration.

There was previous history of left sided nasal obstruction, left nasal mass and nasal discharge of six months duration for which patient was admitted in our ward and underwent nasal polypectomy 1 month before the recent admission. There was no history of headache and inability to close the eye at that time. HPE report of the resected mass was suggestive of granuloma pyogenicum 


\section{CASE REPORT}

with dense lymphocytic infiltration in stroma. The patient came again to us after 15 days of nasal polypectomy with present complaints of mass in left nasal cavity, nasal obstruction, nasal discharge, headache, inability to close both eyes and occasional nasal bleeding for which repeat surgery was done and HPE report was suggestive of olfactory neuroblastoma.

On examination nasal septum was deviated to right and reddish brown mass was present in the left nasal cavity with blood present over it, irregular surface and insensitive to touch. On posterior rhinoscopy, mass was present on left chonae covered with slough. Proptosis of both eyes was present. ( $\mathrm{L}>\mathrm{R})$

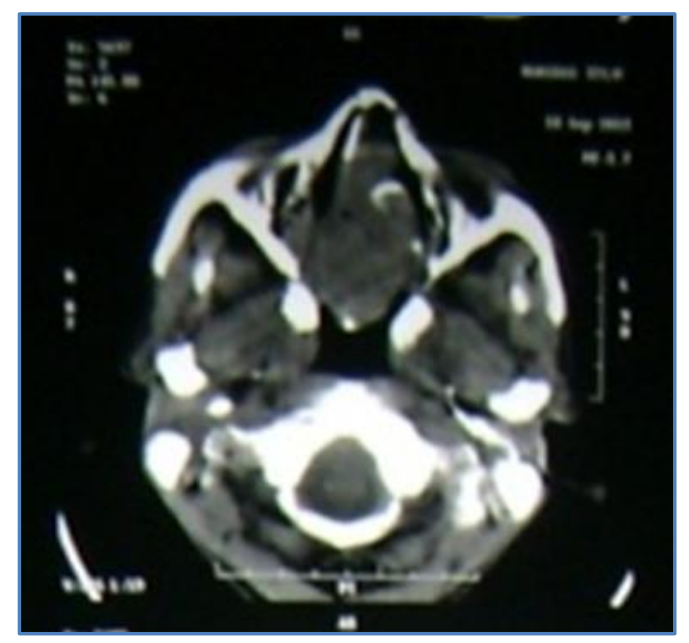

CT Scan

CT finding: Moderate sized, soft tissue density mass lesion in left nasal cavity showing extension into the ipsilateral ethmoid sinus and nasopharynx and causing destruction of nasal septum, ethmoid bone and cribriform plate and showing extension in the right nasal cavity, sphenoid sinus and brain parenchyma.

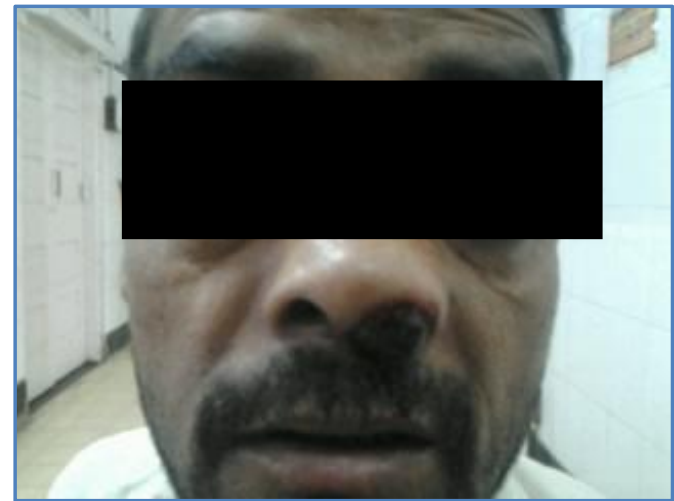

Clinical photograph- pre op

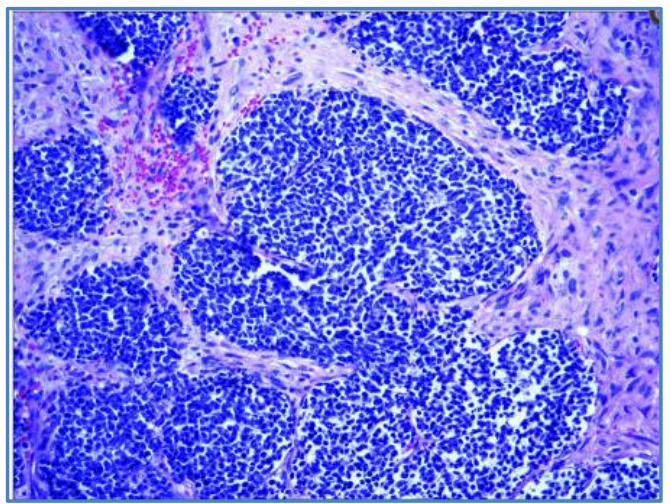

HPE report - Esthesioneuroblastoma 
The tumor consists of large islands of small cells with hyperchromatic nuclei showing a vesicular pattern and scanty cytoplasm. Mitotic activity is brisk and areas of necrosis can be seen. (Hematoxylin and eosin stain).

DISCUSSION: Olfactory neuroblastoma also called as Esthesioneuroblastoma is a relatively uncommon intranasal tumour that originates from the olfactory epithelium of the upper nasal cavity in the region of cribriform plate. ${ }^{3}$

Distant and regional metastasis is present in 14 to $38 \%$ of cases at the time of diagnosis and cervical lymph nodes, lungs and bone are the most commonly involved sites. ${ }^{4}$

The hallmark of olfactory neuroblastoma is the formation of tumour cells in to rosettes, pseudorosettes or sheets and clusters separated by fibrovascular stroma. ${ }^{1}$

Electron microscopy and immunohistochemical studies are frequently needed to establish the diagnosis. Olfactory neuroblastoma stains strongly positive for a variety of neuroendocrine markers such as neuron specific enolase, chromogranin and synaptophysin. The rosettes may be surrounded by several spindle or stellate cells which stains positive for $\mathrm{S}-100 .{ }^{1}$

It is well established that the degree of histopathological differentiation is strongly correlated with the biological behaviour and prognosis. Hyams et al. introduced four grades of differentiation based on growth, architecture, mitotic activity, necrosis, nuclear pleomorphism, rosette formation and fibrillary stroma. A higher grade in the Hyams classification is associated with poorer prognosis.

The grading system is as follows 1 :

Grade 1: tumours have prominent fibrillary matrix, tumour cells have uniform nuclei and lack nuclear pleomorphism, mitotic activity and necrosis.

Grade 2: tumours have some fibrillary matrix, moderate nuclear pleomorphism and some mitotic activity. No necrosis seen.

Grade 3: tumours are characterized by minimal fibrillary matrix and Flexner type rosettes are seen. Prominent mitotic activity, nuclear pleomorphism and some necrosis may be present.

Grade 4: tumours have no fibrillary matrix or rosettes. Marked nuclear pleomorphism and increased mitotic activity seen.

Kadish stages of olfactory neuroblastoma, modified by Morita.

\begin{tabular}{|c|l|}
\hline STAGE & \multicolumn{1}{c|}{ FEATURES: } \\
\hline A & Disease confined to the nasal cavity \\
\hline B & Disease confined to the nasal cavity and paranasal sinuses \\
\hline C & $\begin{array}{l}\text { Disease beyond the nasal cavity and paranasal sinuses, including } \\
\text { involvement of cribriform plate, base of skull, orbit or intracranial cavity }\end{array}$ \\
\hline D & With metastasis to cervical lymph nodes or distant metastasis. \\
\hline
\end{tabular}

A CT scan will assess local invasion in to the adjacent bony structures, particularly the cribriform plate and MR scan will assess the extent of soft tissue invasion, particularly involvement of the anterior cranial cavity and orbit. ${ }^{1}$ 
Craniofacial resection is the standard surgical approach for most cases enabling en bloc resection with the cribriform plate. This has been shown to improve overall survival as well as disease free survival. Five year survival has been shown to improve from 37.5 to $82 \%$ with craniofacial resection. However, despite macroscopic clearance there is relatively high risk of locoregional recurrence of 10 to $30 \%$ and therefore adjuvant therapies with radiotherapy with or without chemotherapy have been explored. ${ }^{1}$

CONSENT: Written informed consent was obtained from the patient for publication of this case report and accompanying images.

\section{REFERENCES:}

1. Stell and Maran's. Textbook of Head and neck surgery and oncology, 5th Edition, Page 792-795.

2. Meneses MS, Thurel C, Mikok J et al. Esthesio neuroblastoma with intracranial extension neurosurgery. 27: 813-820, 1990.

3. Lund VT, Milroy C. Olfactory neuroblastoma: Clinical and pathological aspects. Rhinology. 1993; 31: 1-6.

4. Chan LP, Wang LF, Tai CF, Wu CC, Kuo WR. Huge sphenoid sinus olfactory neuroblastoma: A case report. Kaohsiung J Med Sci. 2009; 25: 87-92.

\section{AUTHORS:}

1. Manish Kumar Sachan

2. A. K. Jain

3. Freni J. K.

4. Kavish Jhawar

\section{PARTICULARS OF CONTRIBUTORS:}

1. Third Year PG Student, Department of ENT, Gajra Raja Medical College, Gwalior, M.P.

2. Professor and HOD, Department of ENT, Gajra Raja Medical College, Gwalior, M.P.

3. Second Year PG Student, Department of ENT,Gajra Raja Medical College, Gwalior, M.P.

4. Second Year PG Student, Department of ENT, Gajra Raja Medical College, Gwalior, M.P.

\section{NAME ADDRESS EMAIL ID OF THE CORRESPONDING AUTHOR:}

Dr. A. K. Jain, Professor and HOD, Department of ENT, Ravi Aakriti Bina Complex, Flat No. G1 and G2,

Gwalior, M.P.

Email: entjainak@hotmail.com

Date of Submission: 09/06/2014. Date of Peer Review: 10/06/2014. Date of Acceptance: 16/06/2014. Date of Publishing: 17/06/2014. 